\title{
The Effect of Product Assortment Changes on Customer Retention
}

\author{
Sharad Borle \\ Jones Graduate School of Management, Rice University, Houston, Texas 77252, sborle@rice.edu \\ Peter Boatwright \\ Tepper School of Business, Carnegie Mellon University, Pittsburgh, Pennsylvania 15213, \\ pbhb@andrew.cmu.edu \\ Joseph B. Kadane \\ Leonard J. Savage University Professor of Statistics and Social Sciences, Carnegie Mellon University, \\ Pittsburgh, Pennsylvania 15213, kadane@stat.cmu.edu \\ Joseph C. Nunes \\ Marshall School of Business, University of Southern California, Los Angeles, California 90089, \\ jnunes@marshall.usc.edu

\section{Galit Shmueli} \\ Robert H. Smith School of Business, University of Maryland, College Park, Maryland 20742, \\ gshmueli@rhsmith.umd.edu
}

\begin{abstract}
$T^{\mathrm{H}}$ his research investigates the impact of a large-scale assortment reduction on customer retention, utilizing a model we develop to explore the effect on sales at both the store level and the category level simultaneously. We apply our model to a data set provided by an online grocer. The data contain detailed household purchase records for every category in the store. Our results indicate that the reduction in assortment reduces overall store sales, a result that contrasts with that of all of the recent studies on assortment reductions (Food Marketing Institute. 1993. Variety or duplication: A process to know where you stand. Prepared by Willard Bishop Consulting and Information resources, Inc., in cooperation with Frito Lay; Drèze, Xavier, Stephen J. Hoch, Mary E. Purk. 1994. Shelf management and space elasticity. J. Retailing 70(4) 301-326; Broniarczyk, Susan M., Wayne D. Hoyer, Leigh McAlister. 1998. Consumers' perceptions of the assortment offered in a grocery category: The impact of item reduction. J. Marketing Res. 35(May) 166-176; Boatwright, Peter, Joseph C. Nunes. 2001. Reducing assortment: An attribute-based approach. J. Marketing 65(July) 50-63; Boatwright, Peter, Joseph C. Nunes. 2004. Correction note for "Reducing assortment: An attribute-based approach." J. Marketing. Forthcoming). We find the reduction had a negative effect on both shopping frequency and purchase quantity, and we find that the decline in shopping frequency resulted in a greater loss than did the reduction in purchase quantities. We also find that the impact of the assortment cut varies widely by category, with less-frequently purchased categories more adversely affected. The variation in the assortment reduction's impact across categories suggests that managers compare select categories in order to moderate the overall loss in sales.

Key words: customer retention; product assortment; efficient assortment; category management; variety; hierarchical Bayes; COM-Poisson

History: This paper was received July 29, 2002, and was with the authors 17 months for 5 revisions; processed by Greg Allenby.
\end{abstract}

\section{Introduction}

Recent research in both the academic and the practitioner literatures suggests grocery retailers can reduce product assortment with little or no loss in sales. A Food Marketing Institute study (1993) found no significant loss in sales after a reduction in the number of stock-keeping units (SKUs) in six test categories across three retail chains, and Boatwright and Nunes $(2001,2004)$ also found no impact on sales in a study of 42 categories. Drèze et al. (1994) found sales actually went up nearly $4 \%$ in eight test categories after experimenters deleted $10 \%$ of the less-popular items. Similarly, a study by Broniarczyk et al. (1998) found that substantial reductions of low-selling or smallshare items can occur without significantly impacting perceptions of assortment within a category.

Offering large assortments is costly, and Wal $*$ Mart's success has forced retailers to reevaluate the current business model. According to the notion of "efficient assortment," cost reductions associated with reducing the number of slow-selling items a grocer carries within a category should result in increased category 
profits. For example, Progressive Grocer (Krum 1994) reported $87 \%$ profit gains in a study of cat box filler where the number of category items was reduced from 26 to 16. Nevertheless, retailers remain reluctant to cut assortments. One of the primary value propositions offered by many grocers is one-stop shopping; customers can find exactly what they are looking for without visiting multiple stores. Retailers fear that backing off from this principle could steer consumers to a competitor that continues to offer a broader product assortment. Simply put, retailers fear assortment reductions erode customer retention.

This research evaluates the outcome of an extensive reduction in assortment conducted by a retailer abiding by the principles of efficient assortment espoused by analysts and supported by prior studies. Specifically, we investigate the impact on customer retention of a large-scale, one-time assortment reduction across each and every category within the store. At the most basic level, we investigate whether or not customers changed their shopping behavior after being offered fewer alternative products from which to choose. The results from our panel-level model indicate that the reduction in assortment reduces overall store sales, and decreases both sales frequency (store visits) and quantity (total basket size). We also find that the assortment reduction had a greater overall effect on purchase frequency than on purchase quantity. The decline in sales is in contrast with previous research suggesting that eliminating moderate amounts of low-selling SKUs results in unchanged or increased category sales (Broniarczyk et al. 1998; Drèze et al. 1994; Boatwright and Nunes 2001, 2004). It is important to point out that earlier research focused on assortment changes within select sets of categories, while we investigate the effects of an assortment reduction on customer retention both across all categories and within individual categories.

Our category-level analysis reveals that lessfrequently purchased categories are more adversely affected by the reduction in assortment than other categories. We also find that the reduction has a greater effect on purchase frequency than on purchase quantity at the category level (category purchase incidence and purchase quantity), just as it did at the store level. Recent empirical studies have focused on exploring the effects of assortment cuts on purchase quantities in popular categories. We suspect this concentration on frequently purchased categories, where our model predicts a decline in purchase frequency would have a less-profound effect, may contribute to the varying results across different studies. It is important to note that sales did not decline in every category, and our results also reveal certain categories for which purchase frequency actually increases, while quantity remains unchanged.
To summarize, the primary purpose of this research is to explore the effect of a large-scale, one-time decrease in assortment on customer retention at both the store and category levels simultaneously. We utilize sales data from each and every category within a store to examine the effect of a reduction in lowselling SKUs on both category and storewide sales. We document that, while the impact was positive in several individual categories, overall store sales decreased, a result not predicted by previous academic or industry research, or by management at the online grocer providing the data.

\section{The Data}

The data come from a natural experiment conducted by an online grocery/delivery service, wherein 840 households (experimental group) were offered a reduced product assortment, while a full assortment was offered to 378 households (control group). The extent of the assortment reduction varied from $24 \%$ to $91 \%$ of items within a category. We utilized all households in the data set that have at least two purchase occasions, resulting in 7,743 experimental-group purchase occasions and 3,275 for the control group. The data for both the experimental group and the control group span January 1997 through January 1998; the assortment was reduced for the last six months of this period. The bulk of purchase amounts (more than $95 \%$ ) lie within the $\$ 40$ to $\$ 220$ band. The interdelivery times range from one week to about 51 weeks.

As for the composition of shopping baskets, we observe purchases in 147 product categories, with the average number of categories purchased per occasion being 20. For our category-level analysis we selected the top 75 categories in terms of number of purchases and combined all remaining categories into one (naming it "all others," a 76 category). The top 75 categories account for more that $90 \%$ of purchases, both in terms of number of purchases and dollars involved. The technical appendix on the Marketing Science website (http://mktsci.pubs.informs. org) contains further details on these categories. ${ }^{1}$

\section{Frequency-Quantity Model}

We develop models of consumer purchase behavior at two levels, the store level and the category level. The "store-level" models are used to study the effects of assortment reductions on a consumer's decisions involving purchase timing (when to shop at the store) and purchase amount (how much to spend for the

\footnotetext{
${ }^{1}$ Due to space constraints, all category-level data, model, and estimation results are included in the technical appendix. However, relevant results from that technical appendix are referenced and discussed in this article.
} 
entire shopping basket). The "category-level" models are used to study the effect of assortment reductions on the category incidence and category purchase amount decisions. At the store level, we model the joint distribution of purchase quantity and timing with reductions in assortment as a covariate. At the category level, we use independent models (conditional on a purchase being made at the store) for category incidence and a joint model (across categories) for the spread of the entire shopping amount across the categories purchased.

\subsection{Store-Level Models}

We observe the interdelivery time $\mathrm{TIME}_{h t}$ in units of days as well as the corresponding purchase quantity $Q_{h t}$ (the entire shopping basket amount) in dollars, where $h$ indexes households and $t$ indexes observations for a household. As in Boatwright et al. (2003), we build the joint model of timing and quantity by considering the marginal distribution of interdelivery time and the distribution of quantity conditional on the interdelivery time.

3.1.1. Interdelivery-Time Model. We aggregate the days to weeks (rounding off to the closest integer week) and assume a COM-Poisson (ComP) distribution for these weeks, thus,

$$
w_{h t} \sim \operatorname{ComP}\left(\lambda_{h t}, \nu\right),
$$

where $w_{h t}=1,2,3, \ldots$ measures the interdelivery time in weeks and $\left(\lambda_{h t}, \nu\right)$ are the parameters of the COM-Poisson distribution. The COM-Poisson is a generalization of the Poisson distribution with an extra parameter $\nu$ that governs tail mass (Boatwright et al. 2003, Shmueli et al. 2004). The probability mass function (p.m.f.) of this distribution is as follows,

$$
P_{z}(\lambda, \nu)=\left[\sum_{k=0}^{\infty} \frac{\lambda^{k}}{(k !)^{\nu}}\right]^{-1} \frac{\lambda^{z}}{(z !)^{\nu}}, \quad z=0,1, \ldots{ }^{2}
$$

The Poisson, the geometric, and the Bernoulli distributions are special cases of the COM-Poisson (with $\nu=1,0$, and $\infty$, respectively).

The parameter $\lambda_{h t}$, given $\nu$, is proportional to the expected interdelivery time, making it a measure of central tendency. We introduce the assortment change effects by specifying $\lambda_{h t}$ as follows,

$$
\log \lambda_{h t}=\lambda_{h}+\delta_{1} \mathrm{ASSORT}_{h t}+\delta_{2} \log Q_{h t-1},
$$

where $\mathrm{ASSORT}_{h t}$ measures the assortment reduction in product categories bought by household $h$ till the $t$ th purchase occasion. It is the natural logarithm of the ratio of the number of unique items (SKUs) available prior to assortment reduction to the number of

${ }^{2}$ We model $z_{h t}=\left(w_{h t}-1\right)$ so that the domain of $z_{h t}$ is $[0, \infty)$.
SKUs available after the assortment reduction in all of the categories bought by the household until purchase occasion $t$. Higher values of the variable indicate a larger assortment cut, and this variable is equal to 0 for any purchase occasion prior to the date of assortment cuts. The variable $Q_{h t-1}$ is one lag of the purchase quantity measured in dollars. Heterogeneity across households is modeled by specifying a Normal distribution for the $\lambda_{h} \mathrm{~s}$,

$$
\lambda_{h} \sim \operatorname{Normal}\left(\bar{\lambda}, \tau^{2}\right),
$$

where $\bar{\lambda}, \tau^{2}$ are, respectively, the mean and variance of the heterogeneity distribution.

3.1.2. Purchase Quantity Model. The purchase quantity is modeled conditional on interdelivery time, as an especially long interdelivery time would likely be followed by a larger order quantity. We assume that

$$
\log \left(Q_{h t}\right) \sim \operatorname{Normal}\left(\mu_{h t}, \sigma^{2}\right),
$$

where $Q_{h t}$ is the purchase quantity in dollars and $\left(\mu_{h t}, \sigma^{2}\right)$ are parameters of the Normal distribution. As with the interdelivery-time model, we incorporate the effects of assortment reduction and interdelivery time by allowing $\mu_{h t}$ to be a function of these variables,

$$
\mu_{h t}=\mu_{h}+\mu_{1} \mathrm{ASSORT}_{h t}+\mu_{2} \log \mathrm{TIME}_{h t} .
$$

Household heterogeneity is modeled by specifying a Normal distribution for the $\mu_{h} \mathrm{~s}$,

$$
\mu_{h} \sim \operatorname{Normal}\left(\bar{\mu}, \theta^{2}\right),
$$

where $\bar{\mu}, \theta^{2}$ are the mean and variance of the heterogeneity distribution. Considering the two store-level models, the parameters $\delta_{1}$ in Equation (3) and $\mu_{1}$ in Equation (6) measure the overall sales response at the store level to assortment cuts, where $\delta_{1}$ corresponds to the effect on purchase frequencies (interdelivery times) and $\mu_{1}$ corresponds to the effect on purchase amounts.

\subsection{Category-Level Models}

Following an approach similar to that of the storelevel models (\$3.1), we build a joint model of category incidence and category amounts by considering: (1) the marginal distribution of the purchase probabilities across categories; and (2) the distribution of the overall shopping-basket amount across categories, conditional on a purchase being made in the category.

The impact of assortment reductions at the category level is modeled by introducing two assortment variables ASSORT $F_{h}^{c}$ and ASSORT $N F_{h}^{c}$. The first is the extent of reduction in assortment from among household $h$ 's favorite items in product category $c$, and 
the second is the reduction from among nonfavorite items. ${ }^{3}$ These variables are defined over the interval $[0,1]$ with 0 indicating no cuts in assortment and 1 indicating a $100 \%$ cut in assortment. By definition, if a household did not face an assortment cut, these variables take the value 0 .

The other covariates we use for the category-level models are the quarterly dummies QTR $1_{h t}^{c}, \mathrm{QTR} 2_{h t}^{c}$, and QTR3 ${ }_{h t}^{c}$ for the first, second, and third quarters, respectively; the natural logarithm of one lag of the purchase amount in a category, $Q_{h t-1}^{c}$; and the natural logarithm of the time elapsed (in days) since the last purchase in that category, $\operatorname{TIME}_{h t}^{c}$. The detailed exposition of the category-level models is provided in the technical appendix at http://mktsci.pubs. informs.org.

\section{The Estimated Coefficients}

The store-level and the category-level models are estimated using four Markov chain Monte Carlo (MCMC) samplers (two each for the store-level and category-level models). The appendix details the priors associated with the two store-level models (the remaining two samplers are discussed in the technical appendix at http://mktsci.pubs.informs.org). The convergence diagnostics were carried out using the Bayesian Output Analysis Program (BOA) Version 0.5.0.

\subsection{The Store-Level Models}

The store-level models are specified by Equations (1), (3), and (4) (the interdelivery-time model); and (5), (6), and (7) (the purchase quantity model). Tables 1 and 2 report the posterior means and standard errors for the store-level parameters.

4.1.1. The Interdelivery-Time Model. These results show that at the overall store level, the assortment cuts led customers to purchase less frequently, for the posterior mass of $\delta_{1}$ is above zero. The reduction in assortment contributed to a $25.0 \%$ average increase in the expected interdelivery times.

Similarly, the estimated value of $\delta_{2}$ is positive, implying that larger amounts bought on the previous occasion tend to delay subsequent purchases. However, this effect is fairly small from a managerial perspective, possibly because households shop elsewhere as well, weakening the correlation between interdelivery time and previous purchase quantity. As for

\footnotetext{
3 "Favorite item" is defined as the set of all items purchased by a particular household prior to assortment reduction. Similarly, "nonfavorite item" is the set of all available items that were not purchased by the household prior to assortment reduction.

${ }^{4}$ We also estimated models with the interdelivery time being distributed Poisson, geometric, and the lognormal, respectively. Similar results as reported here were obtained from these models.
}

\begin{tabular}{lr} 
Table 1 & $\begin{array}{l}\text { Parameter Estimates: Interdelivery- } \\
\text { Time Model }\end{array}$ \\
\hline Parameter & Posterior mean (s.e.) \\
\hline$\nu$ & $0.0614(0.00544)$ \\
$\bar{\lambda}$ & $-0.5334(0.04304)$ \\
$\tau^{2}$ & $0.1246(0.00754)$ \\
$\delta_{1}$ & $0.2231(0.01126)$ \\
$\delta_{2}$ & $0.0344(0.00897)$ \\
\hline
\end{tabular}

the remaining parameters, $\bar{\lambda}$ and $\tau^{2}$ specify the heterogeneity distribution over the $\lambda_{h} \mathrm{~s}$; and the estimate of $\nu$, which is far from 1.0, indicates the Poisson would be a poor model for this data.

4.1.2. The Purchase Amount Model. The parameters $\bar{\mu}$ and $\theta^{2}$ specify the Normal heterogeneity distribution over the $\mu_{h} \mathrm{~s}$. The central $95 \%$ posterior interval of the estimated $\mu_{h} \mathrm{~s}$ is $(3.5206,5.0962)$. This corresponds to a variation of $\$ 33.80$ to $\$ 163.40$ in the base-level expected purchase amounts across households. As in the case of interdelivery times, this indicates the large extent of heterogeneity among consumers in their base-level purchase amounts.

The impact of assortment reductions on purchase amounts $\left(\mu_{1}\right)$ was negative, implying that assortment cuts led households to reduce the overall amounts spent per shopping occasion. However, the magnitude of the effect is less than that for interdelivery times; the assortment cuts contributed to an average decrease of $4.8 \%$ in the expected purchase amounts.

The parameter $\mu_{2}$ is the effect of the natural logarithm of the interdelivery time (in days) on the purchase amounts. As expected, this effect is positive, implying that a delay in purchase is likely to increase the purchase amount on the "next" purchase. However, the effect is marginal; doubling the interdelivery time leads to a mere $5.3 \%\left[\exp \left(\mu_{2} * 0.6931\right)-1\right]$ increase in the expected purchase amounts. Again, the magnitude may be small because online customers also purchase from traditional local retailers.

\subsection{Reconciliation of Results Across Studies}

Our results show that the assortment reduction caused a decline in sales, a result that is robust to changes in prior specification. ${ }^{5}$ This sales decrease is in contrast with many of the earlier studies on the effects of assortment cuts. As a validation check for our model, we reran our model utilizing those categories used in Drèze et al. (1994) and in Boatwright

\footnotetext{
${ }^{5}$ We investigated two alternative prior settings, one that assumed households to be very similar to one another (distributions on $\tau^{2}$ and $\theta^{2}$ were both $\operatorname{IG}(1,10)$ ), and one that assumed households to be very different from one another (distributions on $\tau^{2}$ and $\theta^{2}$ were both IG(1,0.01)).
} 


\begin{tabular}{lr} 
Table 2 & $\begin{array}{l}\text { Parameter Estimates: Store-Level } \\
\text { Purchase Amount Model }\end{array}$ \\
\hline Parameter & Posterior mean (s.e.) \\
\hline $\bar{\mu}$ & $4.3285(0.01939)$ \\
$\theta^{2}$ & $0.1861(0.00875)$ \\
$\sigma^{2}$ & $0.0982(0.00141)$ \\
$\mu_{1}$ & $-0.0503(0.00878)$ \\
$\mu_{2}$ & $0.0751(0.00473)$ \\
\hline
\end{tabular}

and Nunes (2001, 2004). Drèze et al. (1994) used eight categories; they found that aggregate sales went up nearly $4 \%$ after experimenters deleted $10 \%$ of the less-popular SKUs and dedicated more shelf space to high-selling items. We considered equivalent categories from our data and found that the cut in assortments increased the aggregate purchase amounts across these categories (an average increase of 13.3\%), but the interdelivery times also increased (an average increase of $14.2 \%$ ). Although the overall effect on sales nearly cancels out, we find the increase in purchase amounts to be of interest.

Boatwright and Nunes (2001, 2004) found no change in sales, as sales were estimated to increase $1.5 \%$, with a standard error of $1.4 \%$. Boatwright and Nunes $(2001,2004)$ used a different subset of households and a subset of product categories. ${ }^{6}$ When we fit our model to the equivalent categories used in their analysis, our results are similar to theirsdecreased purchase frequencies and unchanged purchase amounts. When we fit our model to their households but include all categories, we obtain our original results (purchase frequencies and amounts both decrease). Our investigation into the categories used by Drèze et al. (1994) and by Boatwright and Nunes $(2001,2004)$ reemphasize our conclusion that the effects of an assortment cut vary widely by category and that store-level effects require looking at a representative sample of, if not all, categories.

\subsection{The Category-Level Models}

The technical appendix at http://mktsci.pubs.informs. org provides the parameter estimates (and a detailed discussion on them) for the category-level models. One critical result at the category level is that a reduction in sales was not uniformly observed across all categories, even though, at the overall store level, the assortment cut led to reduced sales. This result reveals how essential it is to examine all of the categories or an appropriately sized random sample if the objective is to study the impact of assortment reductions on the overall store. The second interesting result is the

\footnotetext{
${ }^{6}$ The source of the data set used by Boatwright and Nunes (2001) was the same as ours; they used a subset of the categories and households.
}

differing impact of assortment cuts on purchase incidence vis-à-vis the purchase quantity. ${ }^{7}$ The assortment cut impacted purchase incidence in about $74 \%$ of categories, while it affected purchase quantity in only $36 \%$ of the categories. This echoes the trend observed at the store level, where the impact of assortment cuts on the total shopping basket was much higher on purchase frequency as compared to purchase amounts. Surprisingly, the assortment reduction led people to buy less often (store retention) rather than to simply buy less (buy missing items elsewhere).

In terms of the effect of assortment cuts on favorite versus nonfavorite items, the effect on purchase incidence for nonfavorites was similar to that for favorites. However, there were many more increases in the quantity (share-of-basket) for nonfavorites than there were for favorites. Cuts in nonfavorites engendered share-of-basket increases for 23 categories while cuts in favorites engendered share-of-basket increases for only 4 categories. Reasons why purchase amounts would increase after nonfavorite items include the elimination of clutter, which brought about a sales increase in Boatwright and Nunes (2001, 2004). In addition, Iyengar and Lepper (2000) demonstrated that choosing among too many items is demotivating and can reduce sales.

\section{Conclusions}

\subsection{Discussion}

In contrast to recent empirical studies, we find a significant reduction in assortment created by eliminating slow-selling items reduces overall store sales. We also find that the assortment cut negatively affected both shopping frequency and purchase quantity, and that the decline in shopping frequency resulted in a greater loss than did the reduction in purchase quantities. The benefits of assortment reductions in earlier studies were hypothesized to occur due to the benefits of simplified shopping (the elimination of clutter or redundant items) that balanced or outweighed a loss of variety. These studies focused exclusively on select categories. As each product category contributes only a relatively small portion to storewide sales, we suspect one reason our results differ from those of prior analyses is because our analysis examines the impact on sales in all categories instead of any particular subset.

Even so, we speculated as to why purchase amounts in the categories studied by Boatwright and Nunes (2001, 2004) remained unchanged, while sales in

\footnotetext{
${ }^{7}$ Purchase quantity at the category level is defined in terms of "share-of-basket," or the percentage share of a particular category in the overall shopping basket. See the technical appendix at http://mktsci.pubs.informs.org for further details.
} 
Figure 1 Bar Graph of Categories, Decreasing in Purchase Frequency

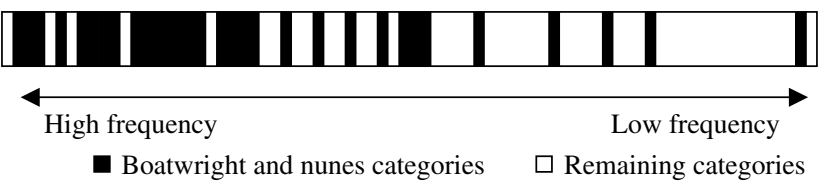

the remaining categories typically decreased. Closer inspection reveals that those categories Boatwright and Nunes (2001, 2004) utilized in their analysis tended to be those of more frequently purchased items, or staples. Consider Figure 1, in which categories are ordered by purchase frequency. The black bars represent the equivalent Boatwright and Nunes (2001, 2004) categories, while white bars represent all other categories. Note how the black bars are concentrated on the left, the high-frequency end of the graph. Also, the first five categories (bars) are produce, milk, bread, cereal, and soft drinks, products that can be thought of as staples. The last five bars are aluminum foil, frozen bakery and whipped toppings, frozen potatoes and onion rings, refrigerated pasta, and the "all others" category-categories not usually considered staples, or regularly purchased goods. Additional analyses confirm the implications of Figure 1 . More frequently purchased categories were less adversely affected in terms of the impact on sales, category incidence, and share of basket. In terms of the magnitude of this effect, for each additional day of category interpurchase time; the percent change in category sales was, on average, an additional loss of $2.23 \%$ (s.e. of $0.71 \%$ ). ${ }^{8}$ Having more items to choose from seems to matter most in categories shoppers visit infrequently. Perhaps they enter these categories with very specific needs, which will not allow substitutions. Or, maybe their inexperience in these categories leads shoppers to require more choice. Our data cannot help us determine the underlying explanation for this effect, which is certainly an area worthy of future research.

\subsection{Future Research and Managerial Implications}

A fruitful avenue for future research would be to further investigate cross-category effects, as reductions in assortments of complementary or competing categories may affect one another. One could also study the impact of assortment changes on the perception of the store itself. A change in assortment may affect some specific segment or segments of consumers patronizing a particular store, permanently altering its customer base, which may become apparent only in the long run, as attrition slowly begins impacting profitability. Another relatively untapped area of investigation involves the number of stores customers

\footnotetext{
${ }^{8}$ See the technical appendix at http://mktsci.pubs.informs.org.
}

visit and their approach to shopping at each, both before and after assortment reductions. Recall the weak correlation between interdelivery time and previous purchase quantity we observed. Managers who provided us with the data also told us that online grocers are only one of a number of stores consumers frequent with regularity, recognizing that shoppers strategically shift their purchases across stores. When and how they do so is certainly worth examining more closely.

One limitation of this study was that the principal experiment was conducted in a single city, in which the delivery fees did not vary. ${ }^{9}$ Data matched to customers' pricing schedules could be used to investigate the degree to which changes in assortment impacted customers under different pricing plans differently, results that could help managers jointly decide assortment levels and delivery charges. Further experimentation could be used to foster more precise prescriptions to managers. For example, an experiment that varies assortment according to types of categories could reveal ways to implement efficient assortment at the store level, because as shown here, category response to changes in assortment are not independent of one another. Nor are they independent of the store itself. Shoppers may forgive the loss of scented toilet tissue, while a loss of variety across all categories may recast the store in an unfavorable light. For instance, that the store may be good for staples but not for bigger trips. Such experiments could reveal the number of categories in which assortment could be reduced without a significant effect on overall store image and visit frequency. In such cases, managers could use some portion of cost savings from efficient assortment in a few categories to promote more frequent store visits and category incidence in those categories where reductions lead to lost sales. Promotions such as loss leaders generate traffic and may be effective in increasing retention at the store level. Promotions such as coupons would be effective at boosting category-specific sales, and these promotions could be tailored to individuals in online environments (Zhang and Krishnamurthi 2004). In addition, further experimentation involving varying degrees of assortment could be used to map out sales response as a function of assortment levels, allowing managers to optimize store profits by adjusting category assortments more precisely (for related work, see Allenby et al. 2004, Dubé 2004, Pauwels and Srinivasan 2004).

\footnotetext{
${ }^{9}$ At the time of the experiment, there were two delivery cost options available to customers. Customers could pay a monthly fee and receive free deliveries, or customers could pay per use. Both assortment and fee schedules impact customer acquisition. Our results do not reflect the impact of assortment on customer acquisition, but changes in usage of existing customers.
} 


\section{Acknowledgments}

The research of Joseph B. Kadane was supported by National Science Foundation Grant DMS-9801401.

\section{Appendix. Prior Specification}

There are four models to be estimated, the two store-level models and the two category-level models. We use a hierarchical Bayes approach using four MCMC samplers (Casella and George 1992, Gelfand and Smith 1990) to estimate these models. The following subsection lays out the prior parameter specifications associated with the two store-level models. Further details on the estimation can be found in the technical appendix at http://mktsci.pubs.informs.org accompanying this article.

\section{A1.1. Prior Specifications}

Table A1 provides the priors used in the estimation. The first store-level model (the interdelivery-time model, §3.1.1) corresponding to Sampler 1 is specified by Equations (1), (3), and (4). Priors are needed over parameters $\nu$ (Equation (1)), $\delta_{1}$ and $\delta_{2}$ (Equation (3)), and $\bar{\lambda}$ and $\tau^{2}$ (Equation (4)). Conjugate Normal and inverse Gamma priors are used for $\bar{\lambda}$ and $\tau^{2}$, respectively, while for $\delta_{1}$ and $\delta_{2}$ Normal priors are used. $\delta_{1}$ specifies the effect of change in assortment on the interdelivery times. A priori there are no strong reasons to believe whether this effect would be negative or positive, i.e., that a reduction in assortment will lead to longer interdelivery times or shorter. Accordingly, we center our prior distribution on $\delta_{1}$ at zero and give it a large variance $(\operatorname{Normal}(0,100))$, representing our little prior knowledge on the parameter. $\delta_{2}$ is the effect of one lag of purchase amounts on the interdelivery times, and one would expect that larger purchases on an occasion may delay the next purchase, although we expect this relationship to be marginal. We use the same prior for $\delta_{2}$ as for $\delta_{1}$. The parameter $\nu$ is defined over the positive real line and, a priori, there is little information on the range of values $\nu$ can take; however, very high values of $\nu$ seem unlikely given the dispersion observed in our data. The prior distribution on $\nu$, a $\operatorname{Gamma}(2,1)$, which has a mode at 1 , is a reasonable representation of our prior beliefs.

The parameters $\bar{\lambda}$ and $\tau^{2}$ determine the cross-sectional heterogeneity distribution of $\lambda_{h}$, which, in turn along with the coefficient for the assortment reduction variable $\left(\delta_{1}\right)$ and the coefficient for the lagged purchase quantity $\left(\delta_{2}\right)$, determines $\lambda_{h t}$, the central tendency parameter of interdelivery time. Again, a priori there is little information to form

\begin{tabular}{lc} 
Table A1 & $\begin{array}{c}\text { The Priors Used in the Estimation } \\
\text { for the Two Store-Level Models }\end{array}$ \\
\hline Parameter & \multicolumn{1}{c}{ Priors } \\
\hline$\nu$ & Gamma $(2,1)$ \\
$\delta_{1}, \delta_{2}$ & Normal $(0,100)$ \\
$\bar{\lambda}$ & Normal $(0,100)$ \\
$\tau^{2}$ & Inverse $\operatorname{Gamma}(2.5,0.5)$ \\
$\sigma^{2}$ & Inverse $\operatorname{Gamma}(2.5,0.5)$ \\
$\mu_{1}, \mu_{2}$ & Normal $(0,100)$ \\
$\bar{\mu}$ & Normal $(0,100)$ \\
$\theta^{2}$ & Inverse $\operatorname{Gamma}(2.5,0.5)$ \\
\hline
\end{tabular}

"sharp" prior expectations for $\lambda_{h}$, thus, we specify priors on $\bar{\lambda}$ and $\tau^{2}$ that can accommodate a wide range of values for $\lambda_{h}$.

The second store-level model (the purchase quantity model, §3.1.2), corresponding to Sampler 2, is specified by Equations (5), (6), and (7). Prior distributions are needed for parameters $\sigma^{2}$ (Equation (5)), $\mu_{1}$ and $\mu_{2}$ (Equation (6)), and $\bar{\mu}$ and $\theta^{2}$ (Equation (7)). Conjugate priors are used for all these parameters; inverse Gamma for $\sigma^{2}$, Normal and inverse Gamma for $\bar{\mu}$ and $\theta^{2}$, respectively, while Normal for $\mu_{1}$ and $\mu_{2}$. As in the interdelivery-time model, $\mu_{1}$ and $\mu_{2}$ specify the effect of assortment reduction and the interdelivery time on the purchase amounts, and $\bar{\mu}$ and $\theta^{2}$ are the parameters of the heterogeneity distribution over $\mu_{h}$. Following similar reasoning as in the interdelivery-time model, we set relatively "diffuse" parameters for these prior distributions. $\sigma^{2}$ is the variance of the purchase quantities (measured on the logarithmic scale) and the inverse Gamma $(2.5,0.5)$ prior on $\sigma^{2}$ is consistent with our lack of specific prior knowledge on this parameter.

\section{References}

Allenby, Greg M., Thomas S. Shively, Sha Yang, Mark J. Garratt. 2004. A choice model for packaged goods: Discrete quantities and quality. Marketing Sci. 23(1) 95-108.

Boatwright, Peter, Joseph C. Nunes. 2001. Reducing assortment: An attribute-based approach. J. Marketing 65(July) 50-63.

Boatwright, Peter, Joseph C. Nunes. 2004. Correction note for "reducing assortment: An attribute-based approach." J. Marketing 68(July) iv.

Boatwright, Peter, Sharad Borle, Joseph B. Kadane. 2003. A model of the joint distribution of purchase quantity and timing. J. Amer. Statist. Assoc. 98 564-572.

Broniarczyk, Susan M., Wayne D. Hoyer, Leigh McAlister. 1998. Consumers' perceptions of the assortment offered in a grocery category: The impact of item reduction. J. Marketing Res. 35(May) 166-176.

Casella, George, Edward I. George. 1992. Explaining the Gibbs sampler. Amer. Statistician 49(4) 327-335.

Drèze, Xavier, Stephen J. Hoch, Mary E. Purk. 1994. Shelf management and space elasticity. J. Retailing 70(4) 301-326.

Dubé, Jean-Pierre. 2004. Multiple discreteness and product differentiation: Demand for carbonated soft drinks. Marketing Sci. 23(1) 66-81.

Food Marketing Institute. 1993. Variety or duplication: A process to know where you stand. Prepared by Willard Bishop Consulting and Information Resources, Inc., in cooperation with Frito-Lay.

Gelfand, Alan E., Adrian F. M. Smith. 1990. Sampling based approaches to calculating marginal densities. J. Amer. Statist. Assoc. 85 398-409.

Iyengar, Sheena S., Mark R. Lepper. 2000. When choice is demotivating: Can one desire too much of a good thing? J. Personality Soc. Psych. 79(December) 995-1006.

Krum, Franklin. 1994. Quantum leap. Progressive Grocer (January) 41-43.

Pauwels, Koen, Shuba Srinivasan. 2004. Who benefits from store brand entry? Marketing Sci. 23(3) 354-390.

Shmueli, Galit, Thomas P. Minka, Joseph B. Kadane, Sharad Borle, Peter Boatwright. 2005. A useful distribution for fitting discrete data: Revival of the COM-Poisson. J. Roy. Statist. Soc., Series C 54(1) 127-142.

Zhang, Jie, Lakshman Krishnamurthi. 2004. Customizing promotions in online stores. Marketing Sci. 23(4) 561-578. 